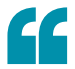

macroketone

inhibits tumour

cell migration and

metastasis by

binding the actin-

bundling protein

fascin 1

METASTASIS

\title{
A FASCINating block
}

Migrastatin - an organic compound naturally occurring in Streptomyces platensis - and its synthetic analogues block tumour cell migration and inhibit metastasis; however, their mode of action is not clear. Xin-Yun Huang and colleagues now report in Nature that the migrastatin analogue macroketone inhibits tumour cell migration and metastasis by binding the actin-bundling protein fascin 1 (FSCN1) and blocking its activity.

In mouse metastatic breast cancer cells, the authors first detected a target protein with a biotinylated protein pulldown assay. Through mass spectrometry and protein sequencing, the protein was identified as FSCN1. Precipitation of glutathione $S$-transferase (GST)-tagged FSCN1 with biotinylated macroketone confirmed a direct interaction between the two molecules. Through fluorescence imaging and electron microscopy, the authors elucidated that macroketone inhibits the formation and thickness of F-actin bundles, suggesting that macroketone blocks the actin-bundling activity of FSCN1. Analyses of the X-ray crystal structures of FSCN1 and the FSCN1-macroketone complex showed that the macroketone-binding site on FSCN1 overlaps with one of the actin-binding sites (see the image). To validate this finding, Huang and colleagues mutated several residues on FSCN1 and investigated the effects on macroketone binding and actin-bundling activity.
The same residues - His392, Lys472 and Ala488 - were essential for both processes, confirming that FSCN1binding sites for macroketone and actin overlap.

However, does macroketone block tumour cell migration and metastasis through FSCN1? Using short hairpin RNA (shRNA) against FSCN1, they demonstrated that the inhibition of actin bundling reduced tumour cell migration and invasion in vitro; expression of wild-type FSCN1 rescued this effect, and the rescued cells were sensitive to macroketone treatment. This suggests that FSCN1 activity is required for the inhibitory action of macroketone. To confirm this hypothesis, they generated a FSCN1 mutant that would selectively bind to actin bundles, but that was resistant to macroketone treatment. Mutagenesis studies revealed that the FSCN1-His474Ala mutant inhibited binding to macroketone, whereas the actin-bundling activity was retained. Importantly, expression of the FSCN1-His474Ala mutant rescued the shRNA-treated cells and restored cell migration. Importantly, macroketone treatment of the mutant-rescued cells did not affect cell migration and invasion, verifying that macroketone inhibits tumour cell migration and metastasis by targeting FSCN1.

Xin-Yun Huang and colleagues went on to test the clinical importance of their findings. In vivo noninvasive bioluminescence imaging

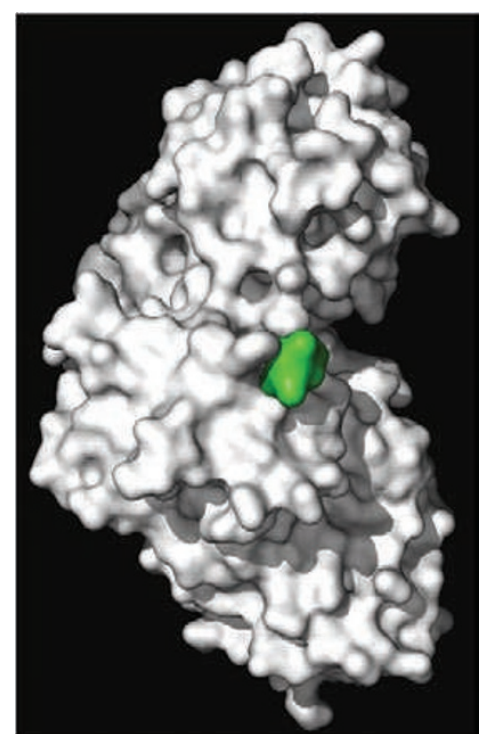

The image shows how macroketone (green) nestles into a pocket (one of the actin-binding sites) of FSCN1. Image courtesy of X.-Y. Huang, Weill Cornell Medical College, New York, USA.

showed that the inhibition of FSCN1 by shRNA or macroketone blocks lung colonization of human breast cancer cells in immune-deficient mouse models. Furthermore, analyses of microarray gene expression data revealed that FSCN1 expression is correlated with oestrogen receptorand progestone receptor-negative breast cancer samples, indicating an association of high FSCN1 expression with a more aggressive clinical course.

These data indicate a role for FSCN1 in tumour cell migration and metastasis and highlight it as a potential drug target for cancer treatment.

Iley Ozerlat

Online Editor, Cell Migration Gateway

ORIGINAL RESEARCH PAPER Chen, L. et al. Migrastatin analogues target fascin to block tumour metastasis. Nature 464, 1062-1066 (2010) 